American Journal of Applied Sciences 5 (4): 418-426, 2008

ISSN 1546-9239

(C) 2008 Science Publications

\title{
Image Recognition Using Combination of Discrete Multi_Wavelet and Wavenet Transform
}

\author{
Dr. Mikhled Alfaouri \\ Department of Communication \& Electronics Engineering \\ Philadelphia University, Amman, Jordan
}

\begin{abstract}
Focus of the method was to present image identification and labeling using new combination of dynamic transforms. This method is based on using the combination of the Discrete Multi-wavelet Transform (DMWT) and Wavenet Transform (WN). In this method, the resulting coefficients were computed by the proposed multi-wavelets transform for single-level decomposition. The low pass sub bands of the upper left corner are considered in the proposed method as a resemblance and a smaller version of the original image. The Wavenet Transform (WN) of low - low coefficients will be obtained one after another and the outcome from the band pass sub bands of the lower right corner is the feature extraction of each image to the recognition identification of the image. This method gave an excellent result: $99 \%$ for a database of 100 different images which indicate that the suggested algorithm is an excellent tool to process the database of standard pose of image. The algorithm is implemented using MATLAB programming languages version 7.
\end{abstract}

Keywords: Discrete Multi-wavelet Transform, Wavenet Transform, Labeling, Low pass, Sub band, Band pass, Recognition.

\section{INTRODUCTION}

This paper provides the dynamic algorithm and step by step method of solution of image identification the systems using the combination of the Discrete Multi-wavelet Transform (DMWT) and Wavenet Transform (WN). This technique is based on "low pass" block (upper left corner) which actually contains one low pass sub band and three band pass sub bands of (L1L1,L1L2,L2L1,L2L2) from the main band of the original image as illustrated in Fig. 1.

Image identification, digital signal processing, computer vision, and image classification are some of the fastest growing areas of computer science and computer engineering. The applications of the above areas in industry, medicine, the military, geology, biology, and other fields, coupled with the progress in computer hardware and software suggest the continual growth of these areas at ever increasing rates. The concern of most researchers in these areas has been to improve the quality of the images and/or to compute some information contained in them with the aim of understanding their content ${ }^{[1]}$.

The proposed transform is considered as a feature extractor of the decomposed reference images with different frequency sub bands, and amid-range frequency sub band for data image to the representation of the given image. Recent works on the methods of Discrete Multi-wavelet Transform (DMWT) and Wavenet Transform (WN) have been focused on the development of the basic theory for image recognition. The combination of this work is based on constructing new multi-filter for de-noising, compressing and recovering the original image. The result obtained by the suggested method is promising and will shed some light to encourage researchers to adopt this technique. Evaluations have generally shown that the technique of the combination for Discrete Multi-wavelet Transform (DMWT) and the Wavenet Transform (WN) is interesting and promising. More theoretical approaches are in need to find a simple and easy algorithm to use for computing Discrete Multi-wavelet Transform (DMWT) and the Wavenet Transform (WN) coefficients.

The Collected Database: In order to take a sufficient database, a total of 100 images were (50 from them to training and another 50 to testing) were collected. The following procedure is used for the data collection:

Step 1: Input the color face image of any size.

Step 2: Give the label for each image as a sequence number 
Step 3: Convert the face image into gray-scale form. Step 4: Resize the image into $128 \times 128$ pixels.

Step 5: Save the resized face image when it is done.

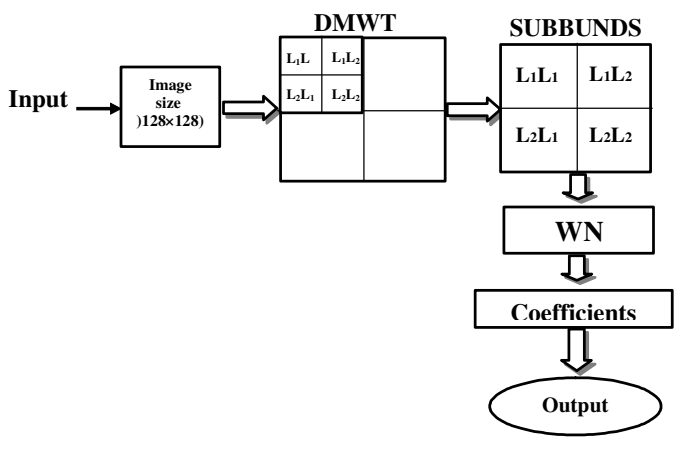

Fig. 1: Main block diagram of the combined transform

The Main Block Diagram of the Proposed Identification Algorithm: Figure1.shows the main block diagram of the algorithm used in the proposed method which consists of the preprocessing method of the Discrete Multi-wavelet Transform (DMWT) and the Wavenet Transform (WN) algorithm. The aim of the preprocessing is to associate the given scalar input signal of length $\mathrm{N}$ to a sequence of length- 2 vector in order to start multi-wavelet transformation process because the given scalar signal consists of one stream of the DMWT algorithm. The results of (L1L1, L1L2, L2L1, L2L2) each of sub band as the input data Wavelet Transform is obtained from them one after another and the result from $\mathrm{L}_{2} \mathrm{~L}_{2}$, sub band is feature extrication for each image.

Multi-wavelet Transform: Wavelets are useful tools for signal processing applications such as image compression and denoising ${ }^{[2]}$. Until recently, only wavelets were known. These are wavelets generated by one scaling function. But one can imagine a situation where there is more than one scaling function. This leads to the notation of multiwavelets, which have several advantages in comparison to wavelets. Such features as short support, orthogonality, symmetry, and vanishing moments are known to be important in signal processing $^{[3]}$. A wavelet cannot possess all these properties at the same time. On the other hand, a multiwavelet system can simultaneously provide perfect reconstruction while preserving length (orthogonality), good performance at boundaries (via linear phase symmetry), and a high order of approximation (vanishing moments). Thus, multiwavelets offer the possibility of superior performance for image processing applications compared with wavelets ${ }^{[2]}$.

One of the important differences between multiwavelets and wavelets is that each channel in the filter bank has a vector-valued input and a vectorvalued output ${ }^{[4]}$. A scalar valued input signal must somehow be converted into a suitable vector-valued signal. This conversion is called preprocessing ${ }^{[2]}$.

The two main categories of methods for doing this are separable and non separable algorithms.

Methods simply work on each dimension in series. The typical approach is to process each of the rows in order, and then process each column of the result. Nonseparable methods work in both image dimensions at the same time ${ }^{[5]}$. While non-separable methods can offer benefits over separable methods, such as savings in computation. They are generally more difficult to implement. Computing Discrete Multi-wavelet Transform, scalar wavelet transform can be written as follows:

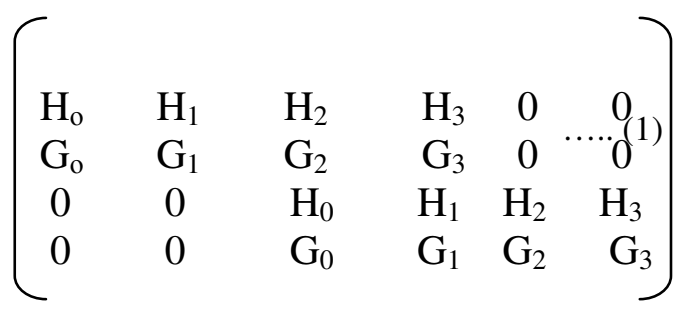

Where $H_{i}$ and $G_{i}$ are low and high pass filter impulse responses, are 2-by-2 matrices which can be written as follows:

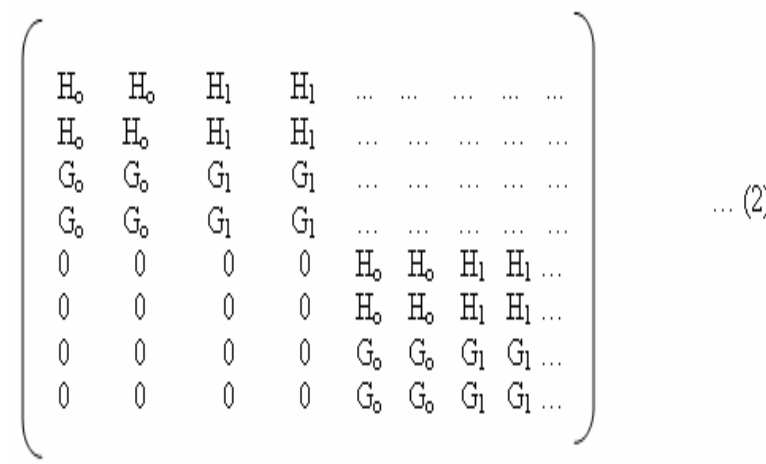

By examining the transform matrices of the scalar wavelet and multi-wavelets, one can see that in the multi-wavelets transform domain there are first and second low-pass coefficients followed by first and second high pass filter coefficients rather than one lowpass coefficient followed by one high pass coefficient. Therefore, if we separate these four coefficients, there are four sub bands in the transform domain. 
Since multi-wavelet decompositions produce two low-pass sub bands and two high pass sub bands in each dimension, the organization and statistics of multiwavelet sub band differ from the scalar wavelet case.

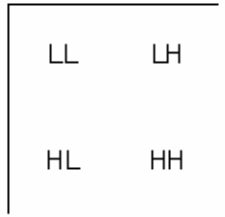

(a)

\begin{tabular}{c|c|c|c|}
\hline $\mathrm{L}_{1} \mathrm{~L}_{1}$ & $\mathrm{~L}_{1} \mathrm{~L}_{2}$ & $\mathrm{~L}_{1} \mathrm{H}_{1}$ & $\mathrm{~L}_{1} \mathrm{H}_{2}$ \\
\hline $\mathrm{L}_{2} \mathrm{~L}_{1}$ & $\mathrm{~L}_{2} \mathrm{~L}_{2}$ & $\mathrm{~L}_{2} \mathrm{H}_{1}$ & $\mathrm{~L}_{2} \mathrm{H}_{2}$ \\
$\mathrm{H}_{1} \mathrm{~L}_{1}$ & $\mathrm{H}_{1} \mathrm{~L}_{2}$ & $\mathrm{H}_{1} \mathrm{H}_{1}$ & $\mathrm{H}_{1} \mathrm{H}_{2}$ \\
$\mathrm{H}_{2} \mathrm{~L}_{1}$ & $\mathrm{H}_{2} \mathrm{~L}_{2}$ & $\mathrm{H}_{2} \mathrm{H}_{1}$ & $\mathrm{H}_{2} \mathrm{H}_{2}$
\end{tabular}

(b)
Fig. 2: Image subbands after a single - level decomposition, for (a) Scalar wavelets and (b) multi-wavelets
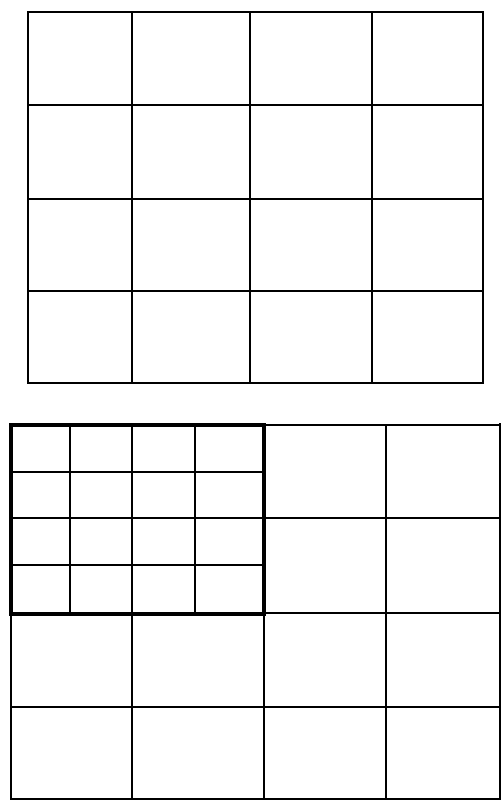

Fig. 3: Conventional iteration of multiwavelet decomposition.

A closer examination of the differences suggests a method for improving the performance of multiwavelets in image applications. During a single level of decomposition using a scalar wavelet transform, the 2D image data is replaced by four blocks corresponding to the sub bands representing either low pass or high pass in both dimensions. These sub bands are illustrated in Fig. (2.a).

The sub band labels in Fig. 2(a) indicate how the sub band data was generated. For example, the data in sub band LH was obtained from high pass filtering of the rows and then low pass filtering of the columns. The multi-wavelets used here have two channels, so there will be two sets of scaling coefficients and two sets of wavelet coefficients. Since multiple iteration over the low pass data is desired, the scaling coefficients for the two channels are stored together ${ }^{[6]}$. Likewise, the wavelet coefficients for the two channels are also stored together. The multi-wavelet decomposition sub bands are shown in Fig. (2b).

For multi-wavelets the $\mathrm{L}$ and $\mathrm{H}$ have subscripts denoting the channel to which the data corresponds. For example, the sub band labeled $\mathrm{L}_{1} \mathrm{H}_{2}$ corresponds to data from the second channel high pass filter in the horizontal direction and the first channel low pass filter in the vertical direction. This shows how a single level of decomposition is done.

In practice, there is more than one decomposition is performed on the image data. Successive iterations are performed on the low pass coefficients from the pervious stage to further reduce the number of low pass coefficients. Since the low pass coefficients contain most of the original signal energy, this iteration process yields better energy compaction ${ }^{[7]}$. After a certain number of iterations, the benefits gained in energy compaction becomes rather negligible compared to the extra computational effort.

Usually five levels of decomposition are used. A single level of decomposition with a symmetric-antisymmetric multi-wavelet is roughly equivalent to two levels of wavelet decomposition. Thus a 3-level multiwavelet decomposition effectively corresponds to 6level scalar wavelet decomposition. The scalar wavelet transform gives a single quarter-sized sub band from the original larger sub band, as shown in figure (2a).

The multi-level decomposition is performed in the same way. The multi-wavelet decomposition iterates on the low pass coefficients from the pervious decomposition, as shown in Fig. (3). In the case of the scalar wavelets, the low pass quarter image is a single sub band. But when the multi-wavelet transform is used, the quarter image of low pass coefficients is actually a $2 \times 2$ block of sub bands (the $\mathrm{L}_{\mathrm{i}} \mathrm{L}_{\mathrm{j}}$ sub bands in Fig. (2b)).

Due to the nature of the preprocessing and symmetric extension method, data in these different sub bands becomes intermixed during iteration of the multiwavelet transform. The intermixing of the multiwavelet low pass sub bands leads to suboptimal results.

Consider the multi-wavelets transform coefficients resulting from single-level decomposition. It can be readily observed that the $2 \times 2$ "low pass" block (upper left corner) actually contains one low pass sub band and three band pass sub bands. The $\mathrm{L}_{1} \mathrm{~L}_{1}$ sub band resembles a smaller version of the original image, which is a typical characteristic of a true low pass sub band. In contrast, the $\mathrm{L}_{1} \mathrm{~L}_{2}, \mathrm{~L}_{2} \mathrm{~L}_{1}$, and $\mathrm{L}_{2} \mathrm{~L}_{2}$ sub bands 
seem to process characteristics more like those of high sub bands. Also only $\mathrm{L}_{1} \mathrm{~L}_{1}$ sub band contains coefficients with a large DC value and a relatively uniform distribution. The $\mathrm{L}_{1}, \mathrm{~L} 1, \mathrm{H} 1$ and $\mathrm{H}_{2}$ sub bands, measured along the vertical direction. Note that $\mathrm{L}_{2}$ sub band looks more like the high pass bands $\mathrm{H}_{1}$ and and $\mathrm{H}_{2}$ than the $\mathrm{L}_{1}$ sub band.

A general procedure for computing DMWT using an Over-Sampled scheme of preprocessing (Repeated Row preprocessing): A general procedure for computing can be made for computing a single-level 2-D DMWT using GHM four multi-filter and using an over-sampled scheme of preprocessing ${ }^{[8]}$.

By using an over-sampled scheme of preprocessing (repeated row preprocessing), the DMWT matrix is doubled in dimension compared with that of the input which should be a square matrix $\mathrm{N}$ x $\mathrm{N}$ where $\mathrm{N}$ must be power of $2^{[9]}$. Transformation matrix dimensions equal image dimensions after preprocessing.

To compute single - level 2-D Discrete multiwavelets Transform, the next steps should be followed:

Checking image dimensions: Image matrix should be a square matrix, $\mathrm{N}$ x N matrix, where $\mathrm{N}$ must be power of 2 .So that the first step of the transform procedure is checking input image dimensions .If the image is not a square matrix some operation must be done to the image like resizing the image or adding rows or column of zeros to get a square matrix ${ }^{[9]}$.

Constructing a transformation matrix: $\mathrm{An} \mathrm{N} \times \mathrm{N}$ transformation matrix should be constructed using GHM low- and high pass filter matrices ${ }^{[11]}$. After substituting GHM matrix filter coefficients values as a $2 \mathrm{~N}$ x $2 \mathrm{~N}$ transformation matrix results with the same dimensions as the input image matrix dimensions after preprocessing will be obtained.

A system with $\mathrm{H}_{\mathrm{k}}$ for $\mathrm{GHM}$ four scaling matrices defined as follows:

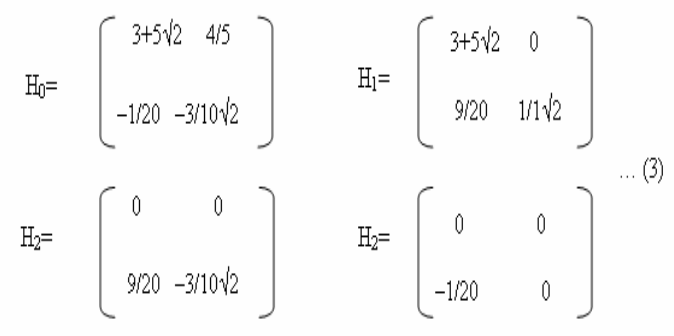

A system with $G_{k}$ for GHM four scaling matrices defined as follows:

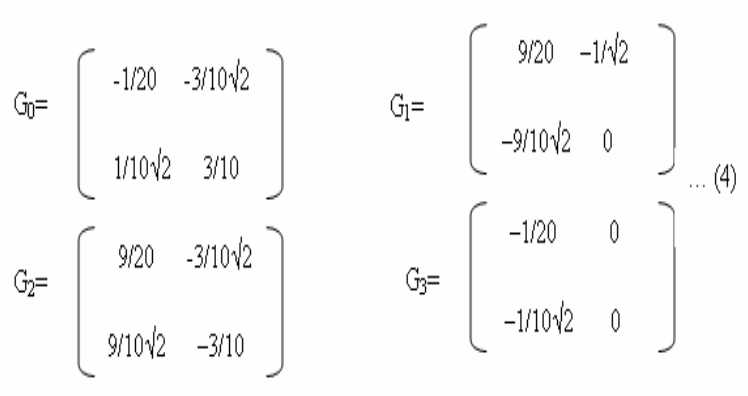

Preprocessing rows: Row preprocessing doubles the number of the input matrix rows. So if the 2-D input is $\mathrm{N} \times \mathrm{N}$ matrix elements, after row preprocessing the result is $2 \mathrm{~N} \times \mathrm{N}$ matrix ${ }^{[7 \& 12]}$. The odd rows $1,3 \ldots 2 \mathrm{~N}$ 1 of this resultant matrix are the same original matrix rows values $1,2,3 \ldots, \mathrm{N}$ respectively. While the even rows numbers $2,4 \ldots 2 \mathrm{~N}$ are the original rows values multiplied by $\alpha$. For GHM system functions $\alpha=1 / \sqrt{ } 2$.

Transformation of image rows: They can be done as follows:

i. Apply matrix multiplication to the $2 \mathrm{~N} \times 2 \mathrm{~N}$ constructed transformation matrix by the $2 \mathrm{~N}$ x $2 \mathrm{~N}$ preprocessed input image matrix.

ii. Permute the resulting $2 \mathrm{~N} \times 2 \mathrm{~N}$ matrix rows by arranging the row pairs 1,2 and $5,6, \ldots, 2 \mathrm{~N}-3,2 \mathrm{~N}-2$ after each other at the upper half of the resulting matrix rows. Then arrange the row pairs 3,4 and $7,8, \ldots, 2 \mathrm{~N}-1,2 \mathrm{~N}$ below them at the next lower half.

Preprocessing columns: It can be done by repeating the same procedure used in preprocessing rows:

i. Transpose the row transformed $2 \mathrm{~N} \times \mathrm{N}$ matrix resulting from step 4

ii. ii. Repeat step 3 to the $\mathrm{N} \times 2 \mathrm{~N}$ matrix (transpose of the row transformed $2 \mathrm{NxN}$ matrix) which results in $2 \mathrm{Nx} 2 \mathrm{~N}$ column preprocessed matrix.

Transformation of image columns: It is applied next to the $2 \mathrm{Nx} 2 \mathrm{~N}$ column preprocessed matrix as follows:

i. Apply matrix multiplication to the $2 \mathrm{Nx} 2 \mathrm{~N}$ constructed transformation matrix by the $2 \mathrm{~N} \times 2 \mathrm{~N}$ column preprocessed matrix

ii. Permute the resulting $2 \mathrm{Nx} 2 \mathrm{~N}$ matrix rows by arranging the row pairs 1,2 and $5,6 \ldots, 2 \mathrm{~N}-3,2 \mathrm{~N}-2$ after each other at the upper half of the resulting matrix rows. Then arrange the row pairs 3,4 and $7,8, \ldots, 2 \mathrm{~N}-1,2 \mathrm{~N}$ below them at the next lower half. 
The final Transformed Matrix: To get the final transformed matrix the following should be applied:

i. Transpose the resulting matrix from column transformation step.

ii. Apply coefficient permutation to the resulting transpose matrix. Coefficient permutation is applied to each of the basic four sub bands of the resulting transpose matrix so that each sub band permutes rows then permutes columns.

Finally, a 2Nx2N DMWT matrix results from the $\mathrm{N} x \mathrm{~N}$ original image matrix by using repeated row preprocessing.

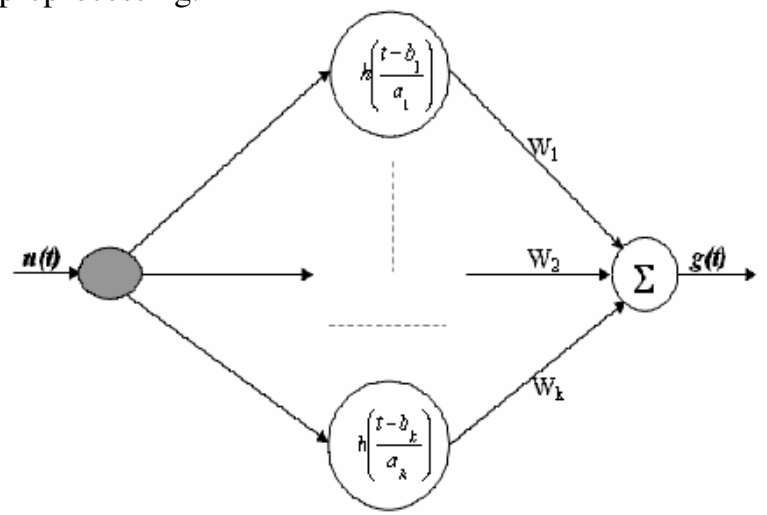

Fig. 4: Adaptive wavenet structure

Wavenet Architecture: In this part, the wavelet transforms theory is combined with the basic concept of neural networks. A new mapping network called, wavenets, is obtained as an alternative way to feed forward neural networks in order to approximate the arbitrary nonlinear functions. Wavenet that uses a special mother wavelet function defined as $\left(h_{a, b}\right)$, is called activation function for Artificial Neural Network (ANN) instead of the traditional function proposed in ${ }^{[12]}$. The wavenet architecture, shown in Figure (4), approximates any desired signal $g(t)$ by generalizing a linear combination of a set of daughter wavelets, where the daughter wavelets are generated by dilation designated by, $a$, and translation designated by, $b$, from a mother wavelet. The choice of mother wavelet depends on the type of signal. If the signal represents a function of one variable, then it will require one variable as a mother function as proposed in ${ }^{[13]}$ :

$$
h_{a, b}(t)=h\left(\frac{t-b}{a}\right)
$$

The signal here is a two dimension Gray-Scale Image (GSI). If the signal represents a function of two variables like images, then it will require twodimensional wavelet filter (mother function):

$$
h_{a, b}\left(t_{1}, t_{2}\right)=h\left(\frac{t_{1}-b}{a}, \frac{t_{2}-b}{a}\right)
$$

where $a$ : Dilation factor of the mother wavelet, with $a>0$.

$b:$ Translation factor.

The approximated signal of the network can be represented by:

$$
g(t)=u(t) \times \sum_{k=1}^{K} w_{k} h_{a_{k}, b}(t) \ldots(7)
$$

Where u (t) is the input signal, $K$ is a number of windowing wavelets, and $w_{k}$ is the weight coefficients, $g, u$, and $h_{a, b}$ may be one single variable or multivariable depending on the input function.

Wavenet Programming Algorithm: In this stage, the proposed algorithm of image approximation is programmed according to the follow procedures:

Step 1: Input the sub band $\mathrm{L}_{1} \mathrm{~L}_{1}$ of the image size $(64 \times 64)$ pixel.

Step 2: Input the initial values of wavelet network parameters, which are:

a. The dilation (a) of the mother wavelet.

b. The weight ( $w)$ of the mother wavelet.

c. Learn rate of weight $(\mu \mathrm{w})$, translation $(\mu \mathrm{b})$, and dilation $(\mu \mathrm{a})$

d. Compute translation (b) new translation = last translation + resolution

Resolution $=$

Upper limit - lower limit

Number of wavelons -1

Step 3: Input the number of wavelons $(\mathrm{N})$, which are required to make image approximation.

Step 4: When the system algorithm reaches the number of iterations which are 7 iterations, the approximated image will be obtained.

Step 5: Store the final dilation, translations and weight set which will be used as an initiation for second sub band of the image.

The Training Phase and Templates Generation: The Novel transformation is proposed to be used for feature 
extraction of the images. The algorithm consists of the following steps:

Step 1: Input the face image into Discrete Multiwavelet Transform (DMWT) proposed Repeated Row Algorithm.

Step 2: A multi-wavelet filter bank requires a vector valued input signal. This is another issue to be addressed when multi-wavelets are used in the transform process, a scalar - valued input signal must somehow be converted into a suitable vector-valued signal. There are many ways to produce such a signal from 2-D image data.

Step 3: Input the image $(128 \times 128)$ matrix, and apply the following steps:

1. Let $\mathrm{X}$ be the input 2-D signal.

2. Matrix input 2-D signal, $X$, and construct a transformation matrix using GHM low- and highpass filters. As GHM filters, $\mathrm{H}$ 's and G's, are $2 \times 2$ matrices. The transformation matrix, $\mathrm{W}$, dimension will be $(2 \mathrm{~N} \times 2 \mathrm{~N})$ after substituting filters coefficients values.

3. Apply row preprocessing to the input 2-D matrix, $X$ by using repeated row preprocessing and $\alpha=1 / \sqrt{ } 2$.

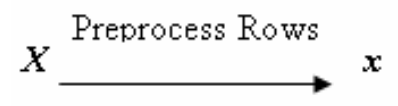

4. Apply row transformation

- Let, $[Z]=[W] \times[x]$

- Permute[Z],

$$
Z \stackrel{\text { Permute }}{\longrightarrow} P
$$

\section{Apply column transformation}

- Transpose $[P]$ matrix.

$$
[P]=[P]^{t}
$$

- Preprocess $[\boldsymbol{P}]^{\mathrm{t}}$ to get $[\mathbf{p}]$.

$$
\text { - } \stackrel{P}{\stackrel{\text { Prebrocess Column }}{\longrightarrow}} \mathbf{p}
$$

- Permute $[\mathbf{b}]$ to get $[\mathbf{B}]$ matrix which is $(128 \times 128)$ matrix.

$$
\text { b } \stackrel{\text { Permute }}{\longrightarrow} \mathrm{B}
$$

6. The final DMWT matrix $[\boldsymbol{Y}]$ results from the following steps,

- Transpose $[\mathbf{B}]$ matrix to get $[\mathbf{y}]$ matrix.

$$
[\mathbf{y}]=[\mathbf{B}]^{\mathrm{t}}
$$

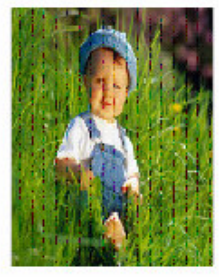

(a)

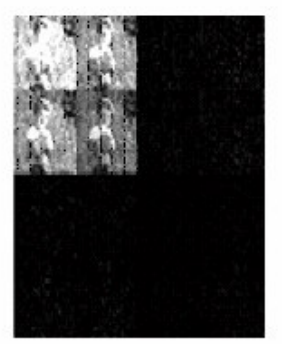

(b)
Fig. 5: Baby image (a) original (b) after single level of DMWT using a repeated row preprocessing

Apply coefficients permutation to each of the four basic sub bands of matrix $[y]$ to get $[Y]$ matrix.

So, $[\mathrm{Y}]$ is the final single-level DMWT matrix. This algorithm is given in Fig. (5).

Step 4: Apply the wavenet transform for each (L1L1, L1L2, L2L1, and L2L2) sub bands. This will result in obtaining twelve coefficients namely four dilations [a's], four translation [b's] and four weights [w's]. The structure of the Wavenet (WN) that was used here is given in Fig. (6).

The computation of wavenet (WN) is carried out by using the following procedure:

a. Set the initial values of a's, b's and w's. Estimate the number of wavelones, boundary of the given sub band $\mathrm{L}_{1} \mathrm{~L}_{1}$ of the first image where the (upper = $64)$, (lower $=1$ ) and fix the learning parameters $\mathrm{M}_{\mathrm{a}}, \mathrm{M}_{\mathrm{b}}$ and $\mathrm{M}_{\mathrm{w}}$.

b. Compute the following intermediate parameters

$$
Z_{1}=\frac{(x-b)}{a}, \quad Z_{2}=\frac{(y-b)}{a}
$$

Where $\mathrm{x}$ is the number of rows and $\mathrm{y}$ is the number of columns.

c. Compute the function $\mathrm{h}\left(\mathrm{Z}_{1}, \mathrm{Z}_{2}\right)$ as below:

$h\left(Z_{1} Z_{2}\right)=Z_{1} Z_{2} e^{-1 / 2\left(Z_{1}{ }^{2}+Z_{2}^{2}\right)}$

d. Obtain the approximated signal and estimate its error with respect to the exact signal. 
e. Repeat the operation until reducing the estimated error below a certain threshold or repeat it for seven times.

Step 5: Arrange the coefficients of wavenet (WN) obtained from step (4) for the given sub band according to the following procedure:

a. Replace $\mathrm{L}_{1} \mathrm{~L}_{1}$ sub band by its 12 wavenet transformed coefficients.

b. The result form the $\mathrm{L}_{1} \mathrm{~L}_{1}$ used the initial values for $\mathrm{L}_{1} \mathrm{~L}_{2}$ to obtain in Wavenet.

Step 6: Repeat step (5) to all LL sub bands of the image and the result form the each sub band used the initial values to anther sub band in the same image.

Step 7: After obtaining $\mathrm{L}_{2} \mathrm{~L}_{2}$ from each image in the Wavenet Transform (WN) the result vector is the feature extraction for each image.

Step 8: Give the label to final vector that result from each image in the Data Base file.

Step 9: Put the final result vector from the last image to initial values for $\mathrm{L}_{1} \mathrm{~L}_{1}$ of another image to obtain in Wavenet (WN).

Step 10: Then repeat stepes $(4,5,6,7,8,9)$ to each image.

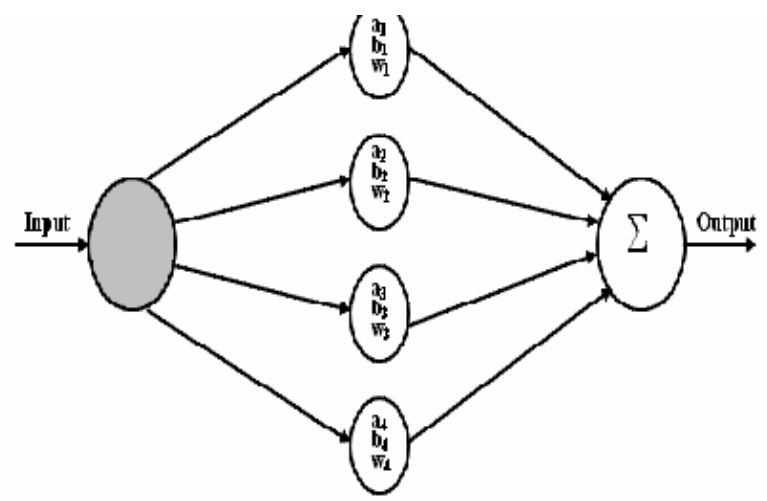

Fig. 6: The implemented structure of the wavenet

It is clear here that the Wavenet (WN) employed here has three types of processing. Firstly it is employed as feature extractor of the segments of the image. Secondly, it works as a data compression techniques in which all the pixels of the image will be represented only by the final 12 coefficients of the latest segment. Thirdly, the final 12 coefficients are used in the classification of the given image with others. Thus, it is employed here as a classifier. Coefficients which is controlled by the number of wavelones obtained.

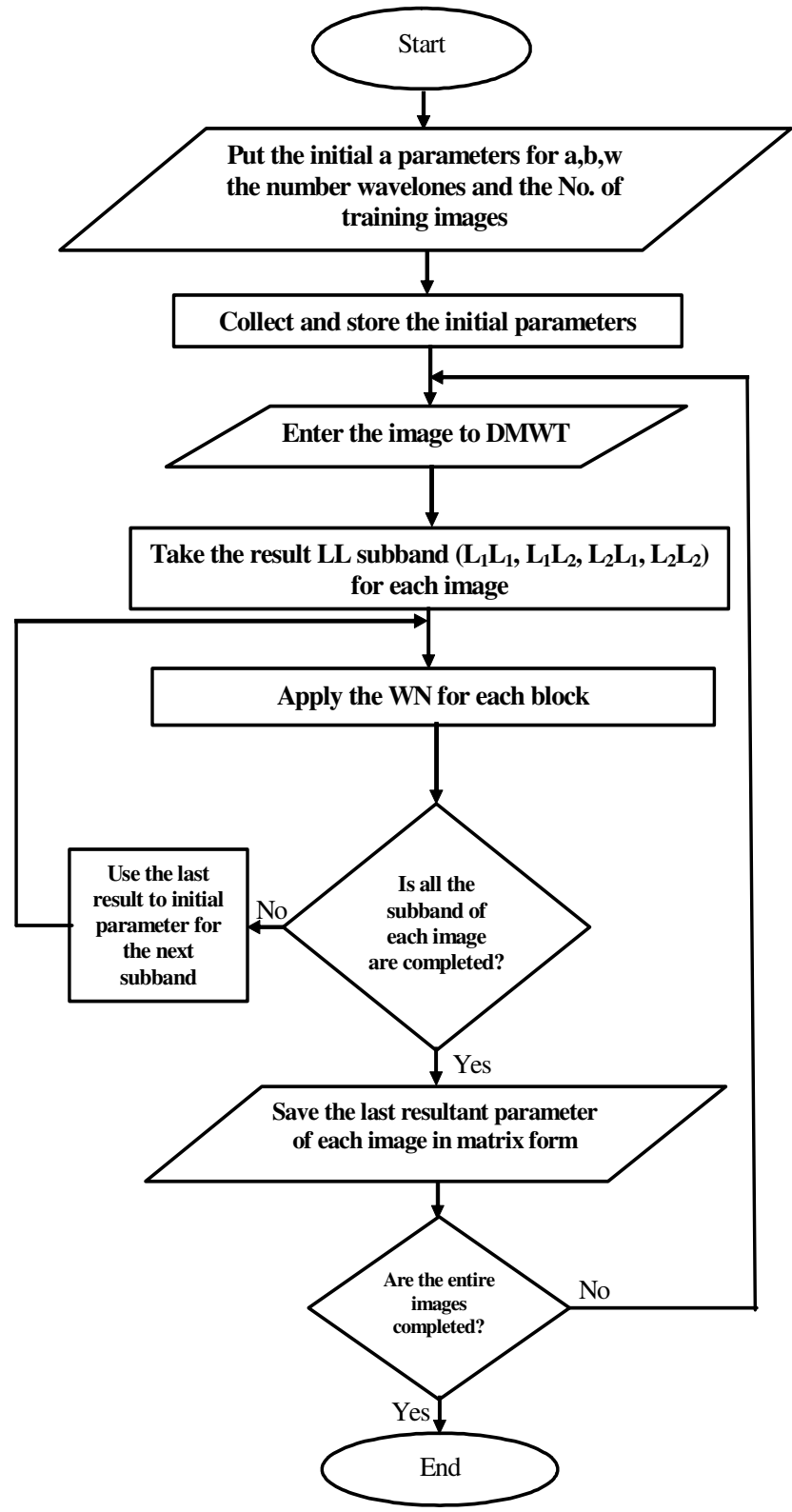

Fig.7: Flowchart for Template Generation phase for each image

The complete structure of this algorithm is shown in Fig. (7).

This is valid because the Wavenet (WN) can handle wide range of data due to its neural network function capability. As well it can accurately represent the data ${ }^{[15]}$. In addition of its capability of representing at randomly samples of each image by only 12 


\section{RESULTS AND DISCUSSION}

In this paper, the results of face image identification depends on using the combination of the Discrete Multi-wavelet Transform (DMWT) and Wavenet Transform (WN). This techniques is based on "low pass" block (upper left corner) which actually contains one low pass sub band and three band pass sub bands from the main band of the original image.

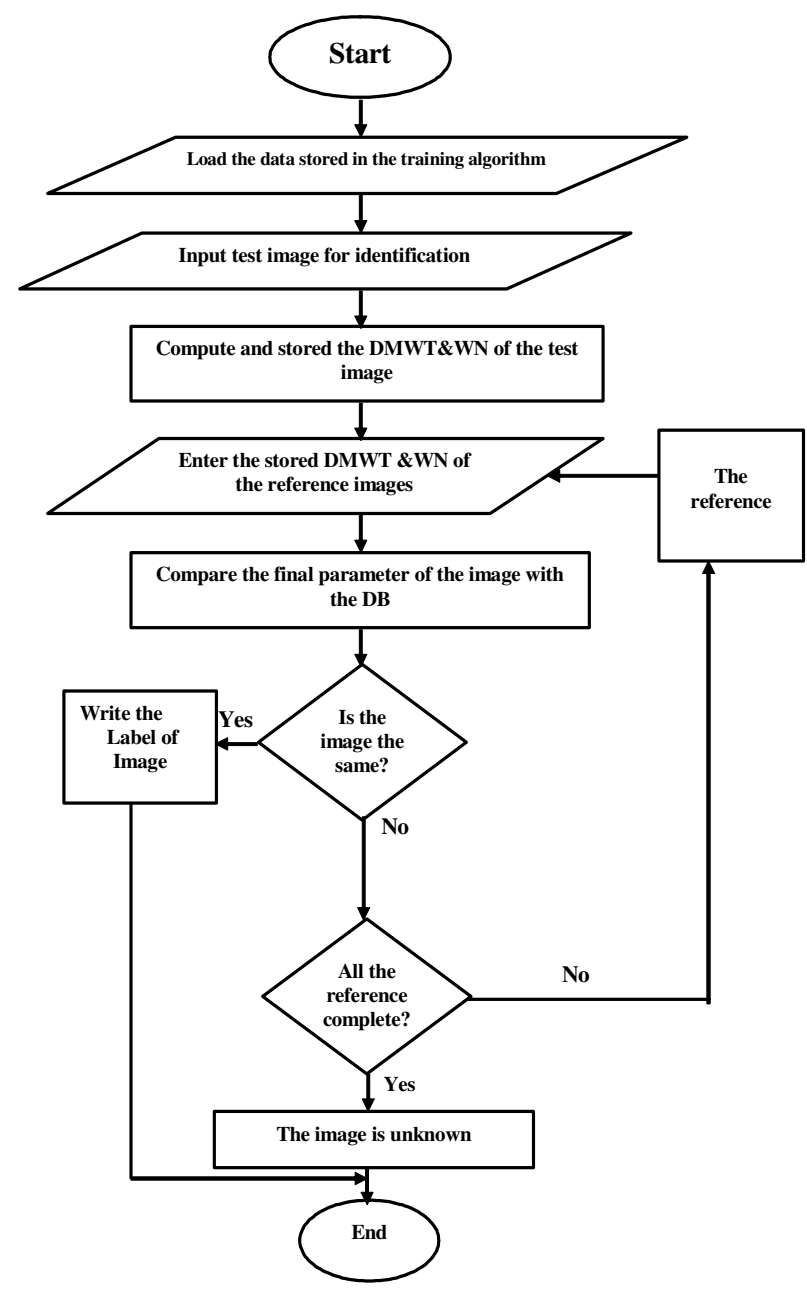

Fig. 8: Proposed combination

Fig. 8 shows how the proposed combination of the Discrete Multi-wavelet Transform (DMWT) and Wavenet Transform (WN) can be used for identification and labeling. It includes the following steps:

Step 1: Obtain the Discrete Multi-wavelet Transform (DMWT) for the test image.
Step 2: Next take the $\left(\mathrm{L}_{1} \mathrm{~L}_{1}, \mathrm{~L}_{1} \mathrm{~L}_{2}, \mathrm{~L}_{2} \mathrm{~L}_{1}, \mathrm{~L}_{2} \mathrm{~L}_{2}\right)$ sub bands result from Discrete Multi-wavelet Transform (DMWT).

Step 3: Then find the Wavenet Transform (WN) to all LL sub bands one after another for each image.

Step 4: Save the result from $\mathrm{L}_{2} \mathrm{~L}_{2}$ of Wavenet Transform (WN) to coefficients for the test image.

Step 5: Finally, compare the corresponding matrix coefficients for the test image with the reference images.

\section{CONCLUSIONS}

It was shown throughout this paper that dynamic transforms had a great impact on the capabilities of all image techniques. Method of image identification was implemented and tested based upon the proposed combination. The advantage of this method which depended on combining the Discrete Multi-wavelet Transform (DMWT) of Repeated Row Algorithm (RRA) and the Wavenet Transform (WN) is summarized in the following:

1. The combined technique achieved good results of 99\%. This was achieved through the suggested combination of the Discrete Multi-wavelet Transform (DMWT) and by using only the (LL) sub bands that were obtained by the Wavenet Transform (WN).

2. This method can give an excellent representation of the data images as well as reduce the huge information obtained as a matrix to a vector one.

3. This dynamic transform is important in many applications particularly when the high data rate is required and when a limited bandwidth or small memory is available.

\section{REFERENCES}

1. Alfaouri, Mikhled 2007. "Novel Techniques for Face Recognition Identification and Labeling", International Journal of Soft Computing, Vol.2, No.1 Pages 129-137

2. Alfaouri, Mikhled 2007. "Image Identification and Labeling Using Hybrid Transformation and Neural Network", i-manager's Journal on Future Engineering \& Technology, Vol.2, No. 4 Pages 5061

3. Strela, V., Heller, P. N., Strang, G., Topiwala, P., and Heil, C., 1999. "The Application of Multiwavelet Filter Bank to Image Processing", IEEE Transaction on Image Processing, Vol. 8, No. 4, PP. 548-563 
4. Geronimo, J., Hardin, D., and Massopust, P. R.,1994. "Fractal Function and Wavelet Expansions Based on Several Functions", J. Approx. Theory, Vol. 78, PP. 373-401

5. Strang, G., and Strela, V., 1995 "Short Wavelet and Matrix Dilation Equations", IEEE Trans. Signal Processing, Vol. 43, PP. 108-115

6. Beegan, A. P. and Bell, A. E., "Noisy Image Compression: A Compression of Wavelets, Multiwavelets, Wavelet Packets, and Multiwavelet Packets", ECE Department, Virginia Tech, Blacksburg, VA

7. Alfaouri, Mikhled and Nada Al-Ramahi, 2007 "New Techniques for Face Image Recognition Identification and Labeling", i-managerm's Journal on Software Engineering, Volum 1, No. 4

8. Strang, G., and Nguyen, T., 1995 "Wavelets and Filter Banks", Wellesley, MA: WellesleyCambridge Press

9. Chui, C. K. and Lian, J., 1996 "A Study on Orthogonal Multiwavelets", J. Appl. Number. Math, PP. 273-298

10. Vaidyanathan, P. P., 1993 "Multirate Systems and Filter Banks", Prentice Hall, Englewood Cli_s, NJ, First Edition
11. Michael B. Martin and Amy E. Bell, 2001. "New Image compression Techniques Using Multiwavelets and Multiwavelet Packets", IEEE Transactions on Image Processing, Vol. 10, No.4

12. Sidney Burrus, Ramesh A. Gopinath, and Haitao Guo, 1998."Introduction to Wavelets and Wavelet Transforms", Prentice-Hell, Inc., Lena Image

13. Contronei M, Lazzaro D, Montefusco L. B, and Puccio L, 2000. "Image Compression Through Embedded Multiwavelet Transform Coding", IEEE Trans. Image Processing, Vol. 9, No. 2: Pages 184 $-189$

14. S. Lawrence, C. Lee 1997."Face Recognition A Convolutional Neural Network Approach", IEEE Transaction on Neural Network. PP 98-113 ,Vol.8

15. R. Chellappa, C. L.Wilson and S. Sirohey, 1995. "Human and Machine Recognition of Face": a Survey, Proceedings of the IEEE, Vol. 83, No. 5: Pages $705-740$. 